\title{
CARACTERIZAÇÃO E VARIABILIDADE INTERANUAL DA PRECIPITAÇÃO PLUVIOMÉTRICA NA ESTAÇÃO ECOLÓGICA DO TAIM, RIO GRANDE DO SUL, NO PERÍODO DE 1996 A 2009.
}

\author{
SIMIONI, João Paulo Delapasse - jpaulosimioni@hotmail.com \\ Graduando do Curso de Geografia - Universidade Federal de Santa Maria \\ WOLLMANN, Cássio Arthur - cassio_geo@yahoo.com.br \\ Geógrafo / Prof. Dr. Universidade Federal de Santa Maria
}

\begin{abstract}
RESUMO: O objetivo geral desta pesquisa consistiu em analisar a variabilidade interanual da precipitação pluviométrica na Estação Ecológica do Taim, no período de 1996 a 2009. Para a realização desta pesquisa utilizou-se os dados de precipitação pluviométrica, obtidos gentilmente junto a administração da ESEC Taim, durante a serie temporal de 1996 a 2009, com exceção do ano de 1999, devido a inexistência de dados neste período. No atlas climático do Rio Grande do Sul, coletaram-se dados das normais climatológicas das estações oficiais do INMET de Santa Vitoria do Palmar e Rio Grande, para fins de comparação. A manipulação, análise estatística e criação dos gráficos dos resultados obtidos foram realizadas no Excel 2010. A variável precipitação pluviométrica demonstrou ser bem distribuída sazonalmente, sendo que os meses de abril e agosto destacam-se pela maior média mensal com $116 \mathrm{~mm}$ e $118 \mathrm{~mm}$, respectivamente. Ao comparar os dados da ESEC Taim com os dados das Estações meteorológicas do INMET verificou-se que no verão o índice de precipitação pluviométrica é menor no Taim se comparado ao das estações das cidades vizinhas, no entanto no outono este valor se inverte e o Taim apresenta um maior regime pluviométrico.
\end{abstract}

PALAVRAS-CHAVE: Precipitação pluviométrica; variabilidade; Estação Ecológica do Taim; Rio Grande do Sul.

CHARACTERIZATION AND INTERANNUAL VARIABILITY OF RAINFALL ON TAIM ECOLOGICAL STATION, RIO GRANDE DO SUL, THE PERIOD 1996 TO 2009.

ABSTRACT: The overall objective of this research was to analyze the interannual variability of rainfall in the Taim Ecological Station, in the period 1996-2009. For this research we used the data of rainfall, kindly obtained with the administration of ESEC Taim, during the time series from 1996 to 2009, except for 1999, due to lack of data at this time. The climate atlas of Rio Grande do Sul, data was collected from climatological normals of the official stations INMET Santa Vitoria do Palmar and Rio Grande, for comparison purposes. The manipulation, statistical analysis and creation of graphs of results were performed in Excel 2010. The rainfall variable shown to be well distributed seasonally, with the months of April and August are distinguished by highest monthly average of $116 \mathrm{~mm}$ and $118 \mathrm{~mm}$, respectively. When comparing data from ESEC Taim with data from meteorological stations INMET it was found that in summer the rate of rainfall is lowest in Taim compared to the stations of the neighboring towns, however in autumn this value is inverted and Taim presents greater rainfall.

KEYWORDS: Rainfall; variability; Estação Ecológica do Taim; Rio Grande do Sul.

\section{INTRODUÇÃO}

No Brasil, os estudos climáticos relacionados às unidades de conservação ainda constituem-se uma minoria dentro da seara geográfica, mesmo com a criação do Sistema Nacional de Unidades de Conservação (SNUC), regulamentado pela Lei Federal No 9.985, de 18 de julho de 2000, que em seu 
Artigo $50^{\circ}$, dispõe que o clima também é considerado uma das características marcantes de uma região, e por tal razão, merece cadastro e proteção dos órgãos ambientais nas esferas federal, estaduais e municipais competentes (WOLLMANN; SIMIONI, 2013).

Assim, estudos temporais das variáveis climatológicas demonstram-se cada vez mais importantes para o entendimento e caracterização do tempo ao longo dos anos em diversas áreas, pois os impactos ambientais e as anomalias climáticas conduzem para um caminho de grande diversidade climatológica através de alterações no clima regional.

Em si, a variável precipitação é um instrumento de suma importância na caracterização do clima, a qual, segundo Bezerra et. al. (2004, 2010); Aguiar (1995); Marengo (2001) interfere em todos as frações das atividades econômicas e na relação do ecossistema através do ciclo hidrológico, pois a água encontra-se em todos os estágios ou fases no sistema superfícieatmosfera.

Para a Estação Ecológica do Taim (ESEC Taim), localizada no extremo sul do Estado do Rio Grande do Sul, o clima e sua dinâmica regional passa a ser um atributo marcante para a vida nesta unidade de conservação, que ora apresenta-se alagada, ora seca, com chegadas e partidas de movimentos migratórios de várias espécies. Nesse sentido, entender a relação existente entre a dinâmica do meio atmosférico e do meio biótico e terrestre é, portanto, um dos objetivos da Climatologia Geográfica.

Dessa forma, esta pesquisa procurará analisar os eventos pluviométricos ocorridos na sede da ESEC TAIM, de modo a contribuir modestamente com estudos científicos dessas duas naturezas (clima e preservação), cuja frágil relação pode ser afetada tanto pelas atividades humanas, quanto pelas dinâmicas naturais.

O Objetivo geral desta pesquisa consiste na análise e caracterização temporal das precipitações pluviométricas na Estação Ecológica do Taim entre 1996 e 2009, com exceção do ano de 1999, o qual não possui dados coletados e registrados.

\section{LOCALIZAÇÃo E BREVE CARACTERIZAÇÃo DA ÁREA DE ESTUDO}

A Estação Ecológica (ESEC) do Taim foi criada através do Decreto Federal n० 92.963, de 21 de Julho de 1986, e segundo o Sistema Nacional de Unidades de Conservação (SNUC, 2000 apud WOLLMANN; SIMIONI, 2013) é classificada como unidades de conservação de Proteção Integral, cujo Artigo 20, Parágrafo VI coloca que sua principal função é a "manutenção dos ecossistemas 
livres de alterações causadas por interferência humana, admitido apenas o uso indireto dos seus atributos naturais".

Localizada no extremo sul do Brasil, no Estado do Rio Grande do Sul (Figura 01), na Planície Litorânea, com altitudes não superiores a 20 metros acima do nível do mar, é considerada, pelo Ministério do Meio Ambiente (MMA, 2011) a Unidade de Conservação Federal mais ao Sul do território brasileiro, e segundo a Fundação Zoobotânica do Estado do Rio Grande do Sul (FZBRS, 2012), a ESEC localiza-se dentro do Bioma Pampa, em litoral lagunar, mas em duas ecorregiões com vegetação distintas: os Campos Sulinos e a Vegetação de Restingas da Costa Atlântica.

Diante dessa variedade ambiental podem ser encontradas várias espécies de animais, tais como o João-de-barro, tartarugas, tuco-tuco, capivaras, ratão-do-banhado, jacaré-de-papo-amarelo e uma abundante avefauna (FLOOR, 1999). Em relação ao clima, a ESEC Taim está localizada na porção menos chuvosa do Rio Grande do Sul (SARTORI, 1993; ROSSATO, 2011; WOLLMANN, 2011), com grande variação térmica (amplitude) anual e é a área do Estado mais afetada pela Corrente Marítima Fria das Falklands, além das passagens semanais de Ciclones Extratropicais (PANCOTTO, 2007) que conferem à região, especialmente no inverno, o clima mais frio e hostil do Estado.

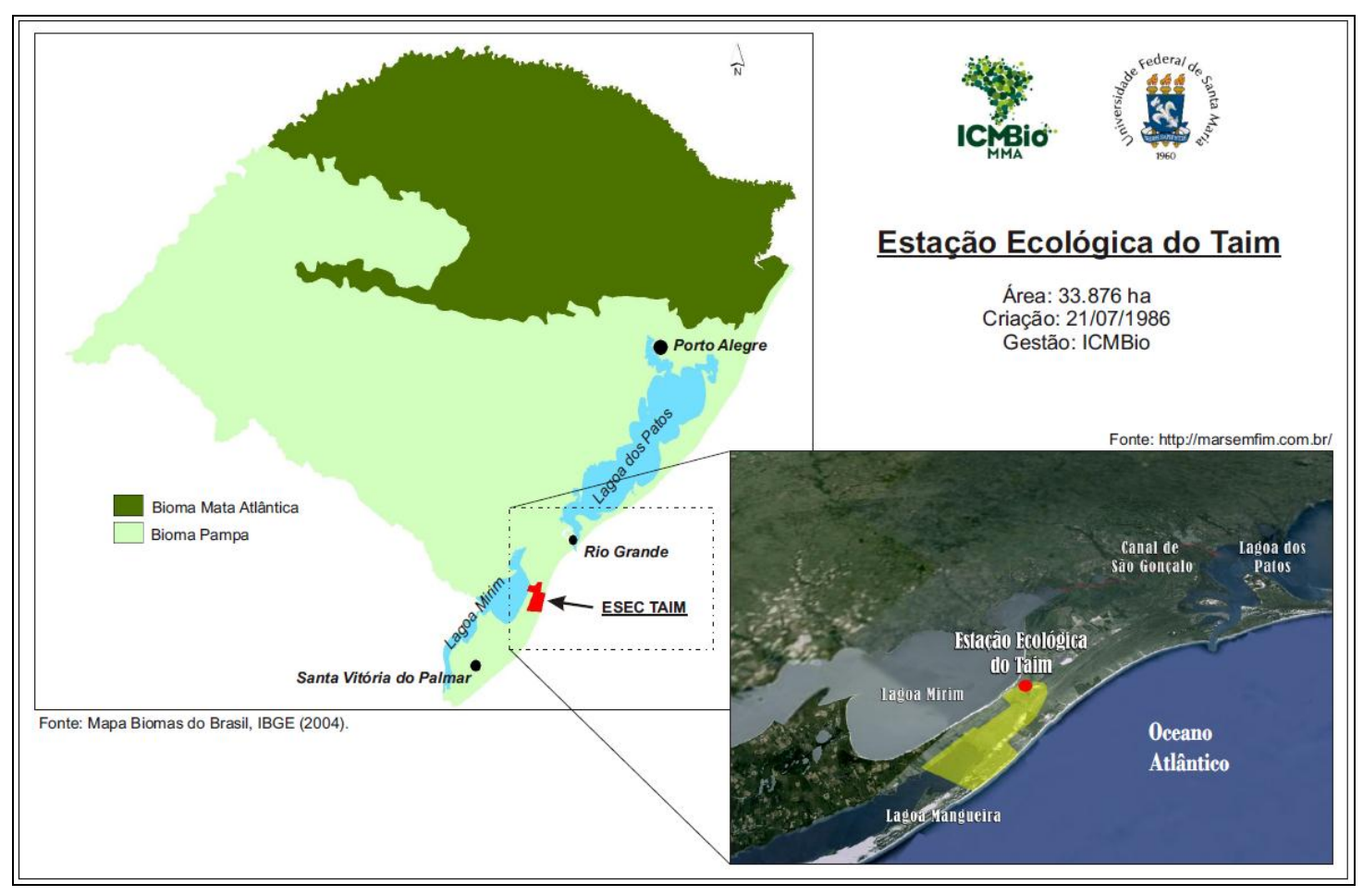

Figura 1 - Localização espacial da ESEC Taim. Adaptação: Simioni; Wollmann (2014). 
Do ponto de vista socioeconômico, a região destaca-se pela sua grande preservação ambiental, mas possui o desenvolvimento das atividades rizícolas e silvicultoras, além da ligação direta com o Uruguai, através da BR 471 que liga Pelotas e Rio Grande a Santa Vitória do Palmar e Chuí, e que atravessa a ESEC. Os limites oficiais da ESEC Taim estendem-se por dois municípios: Rio Grande e Santa Vitória do Palmar (repartindo, mais ou menos, $30 \%$ e $70 \%$ de seu território para cada município, respectivamente), e entendendo-se por uma área de 33.815 hectares. Ainda, atividades turísticas em balneários (Hermenegildo e Cassino), além do Porto de Rio Grande constituem-se nos principais impactos nas áreas do entorno da ESEC, mas localizados a mais de dez quilômetros dos limites legais da estação.

\section{METODOLOGIA}

Para desenvolvimento desta pesquisa foi utilizado dados de precipitação anual do período de 1996 a 2009 da estação climatológica da ESEC TAIM, localizada a 07 metros do nível do mar, com coordenadas 32³2'16.96"S e $52^{\circ} 32^{\prime} 21.55^{\prime \prime O}$ fornecidos gentilmente pela administração da ESEC TAIM.

A metodologia utilizada foi empregada anteriormente em um valoroso trabalho por Bezerra et. al. (2010) e visa calcular estatisticamente a distribuição de frequência diária, mensal, trimestral e anual, tendência central, como média aritmética, mediana, dispersão, amplitude e desvio padrão da precipitação pluvial na ESEC Taim durante o período de 1996 a 2009.

Deste modo as médias aritméticas e o respectivo desvio padrão (s) foram calculados utilizando equações estatísticas como a mediana, e para dados agrupados, utilizaram-se as frequências acumuladas (Figura 2).

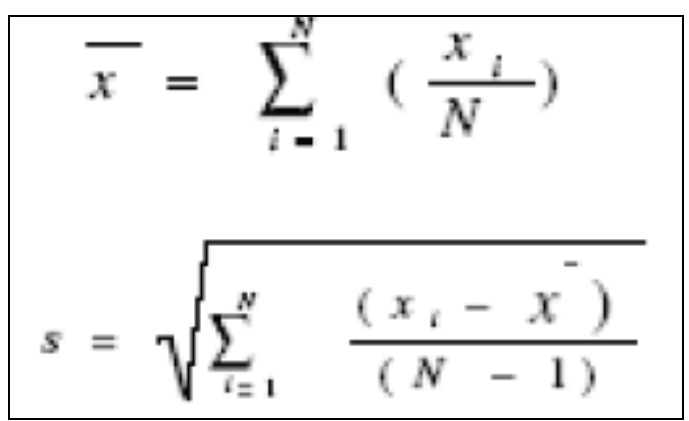

Figura 2 - Equações estatísticas de mediana e frequências acumuladas

Onde xi é o total mensal ou anual de chuva $(\mathrm{mm})$ e $\mathrm{i}=$ número de observações $(1,2,3, \ldots, \mathrm{N})$ 
Para a efetivação destas analises ordenaram-se os dados anuais de chuvas em ordem crescente, sendo as probabilidades empíricas determinadas mediante a equação da Figura 3.

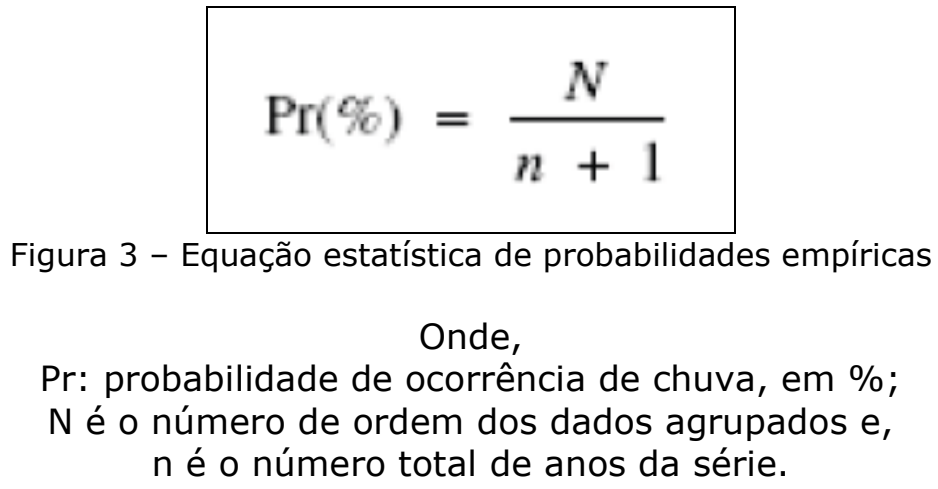

\subsection{Medidas de Dispersão}

As medianas de dispersão representam a dispersão ou a variabilidade dos dados em torno do valor central, a média mais a amplitude que é a diferença entre os valores extremos. Para este estudo será empregado o mesmo método de Bezerra et. al. (2010) onde são utilizados medidas de dispersão para a utilização da variabilidade anual da precipitação anual, o desvio padrão, a variância e o coeficiente de variância (Figura 4).

$$
\begin{gathered}
C V=\frac{S}{\bar{X}} \\
\mathrm{~S}^{2} \rightarrow \text { variância amostral }
\end{gathered}
$$

Figura 4 - Equação estatística de dispersão (desvio padrão, a variância e o coeficiente de variância)

Os desvios de precipitação foram obtidos da subtração da média dos doze anos de precipitação pela precipitação anual da série temporal que foi de 1996 a 2009 (exceto 1999) ano a ano, gerando desvios positivos e negativos, dada pela equação $\mathbf{D P}=\mathbf{P A}-\mathbf{P M}$, onde DP é o desvio de precipitação, PA é a precipitação anual e PM é a precipitação média em planilha do Excel 2010.

\section{ANÁLISE DOS RESULTADOS E DISCUSSÕES}

\subsection{Precipitação Anual}

A precipitação total anual é percebida variando em parcelas de intervalos temporais (Figura 5). Estes intervalos possuem pouca variação de precipitação e, são visíveis entre os anos de 1997 a 2002, sendo uma média aproximada de $1400 \mathrm{~mm}$ de precipitação nestes anos, média esta que é relativamente alta se 
comparado com os demais anos estudados. Este fato deve-se evento de El niño observado no ano de 1997 (COSTA et. al., 2007) o qual provocou um aumento da precipitação média anual destes anos.

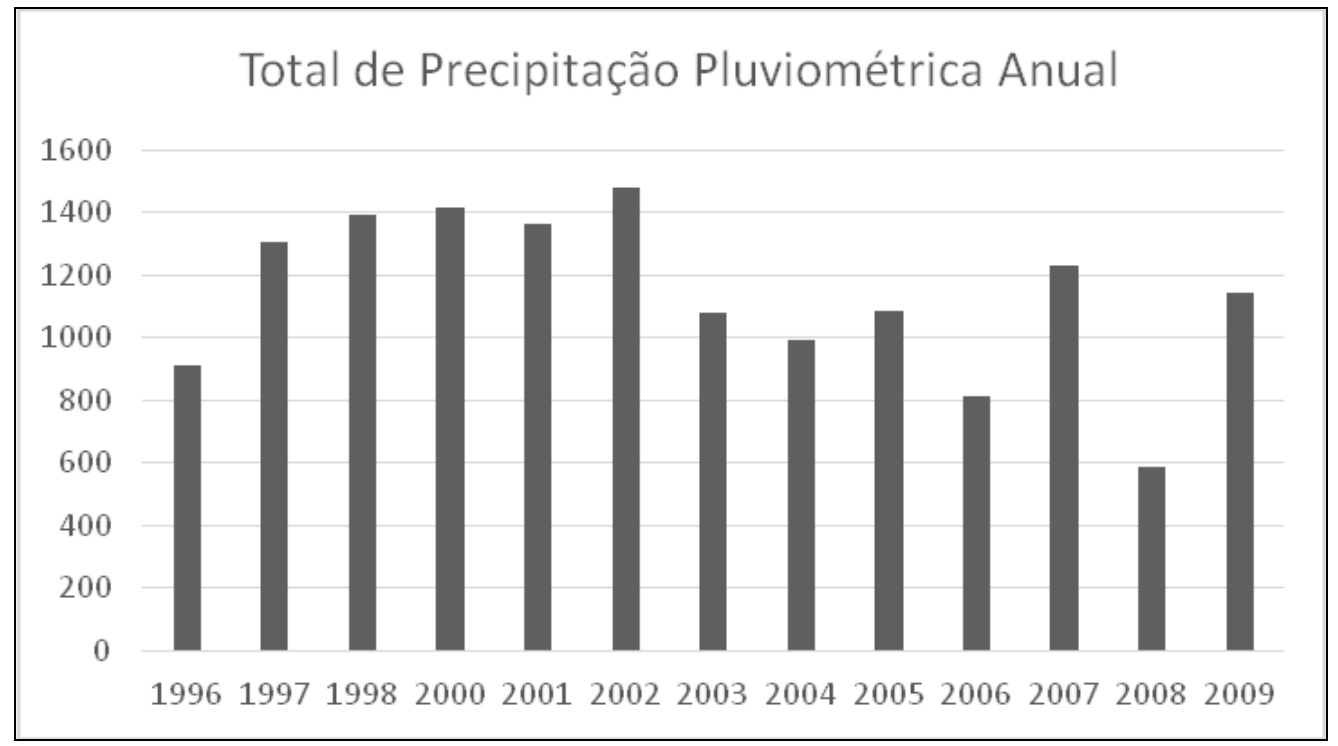

Figura 5 - Precipitação Pluviométrica total anual. Org.: Simioni; Wollmann (2014).

Por outro lado, é possível identificar também um baixo regime pluviométrico associado aos anos conseguintes de 2003 a 2007 onde a média anual de precipitação aproximou-se de $900 \mathrm{~mm}$ anuais. Este fato é associado à 2003 ser um ano neutro (COSTA et. al. op. cit.) o que faz com que as precipitações tenham uma média anual relativamente baixa se comparado ao resto do Estado pois, esta região está inserida na porção menos chuvosa do Rio Grande do Sul (SARTORI, op. cit.; WOLLMANN, op. cit.), com grande variação térmica (amplitude) anual.

\subsection{Precipitação Média Trimestral}

A distribuição da precipitação trimestral é um bom método de análise do comportamento de concentração do regime pluviométrico anual de uma localidade de acordo com BEZERRA et. al. (2010), NOBRE E MELO (2001) e VAREJÃO SILVA (2001), pois a mesma mostra os trimestres de maior e menor concentração de chuva. A figura 6 indica que a precipitação média anual entre os trimestres mais e menos chuvosos comporta-se de modo oscilatório, com 
formato da letra $M$, sendo o primeiro e o ultimo trimestres do ano os de menos ocorrência de precipitação pluviométrica.

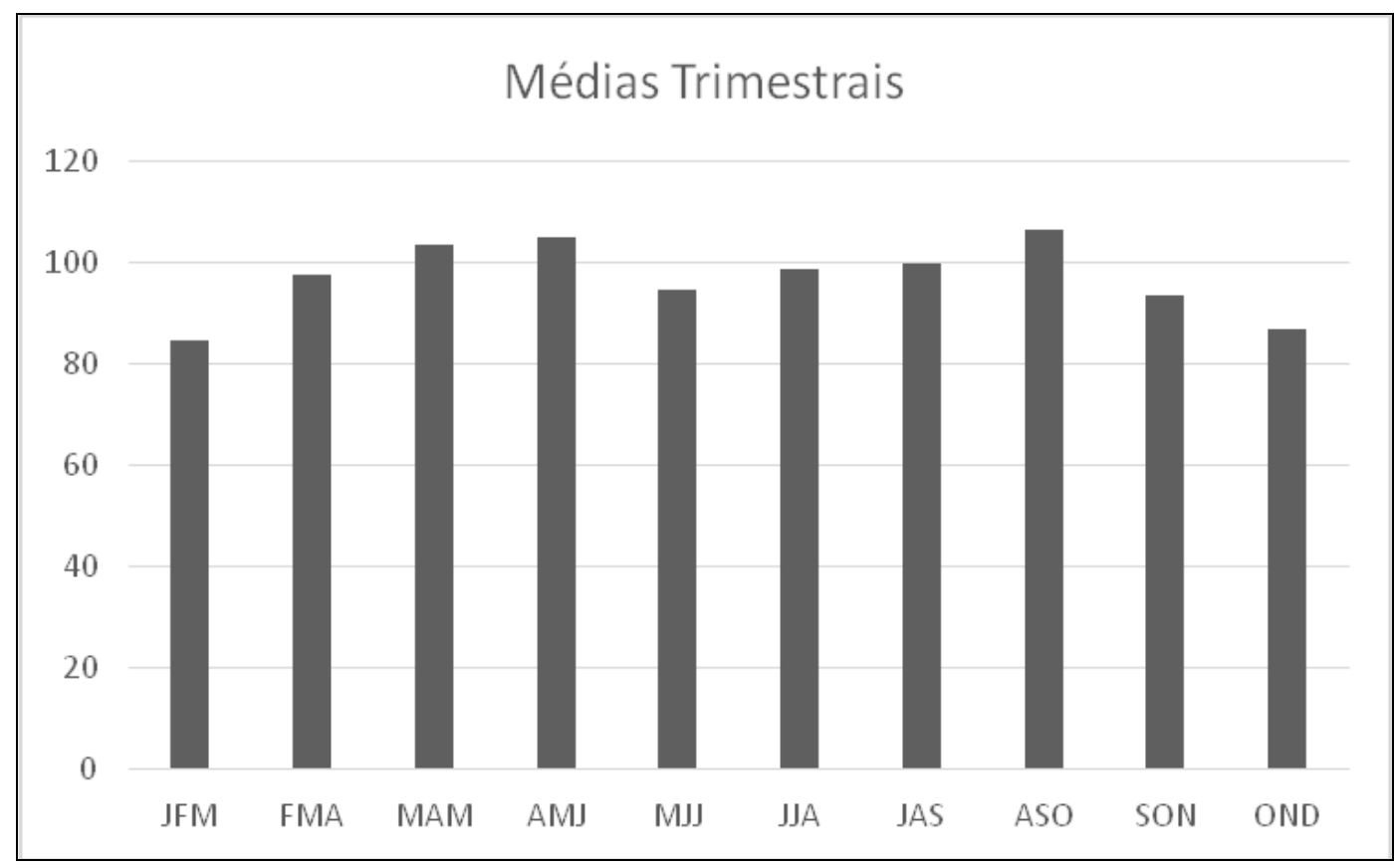

Figura 6 - Precipitação média trimestral na ESEC Taim 1996/2009. Org.: Simioni; Wollmann (2014).

\subsection{Precipitação Mensal}

As principais características da precipitação média mensal na ESEC Taim é sua sazonalidade bem distribuída durante todos os meses, sendo que o mês de agosto e abril destaca-se pela maior média mensal com $118 \mathrm{~mm}$ e $116 \mathrm{~mm}$ respectivamente (figura 7 ). Os meses de menor pluviosidade são percebidos na estação de verão, onde, novembro apresenta $78 \mathrm{~mm}$, dezembro $79 \mathrm{~mm}$ e janeiro $74 \mathrm{~mm}$ de médias mensais. 


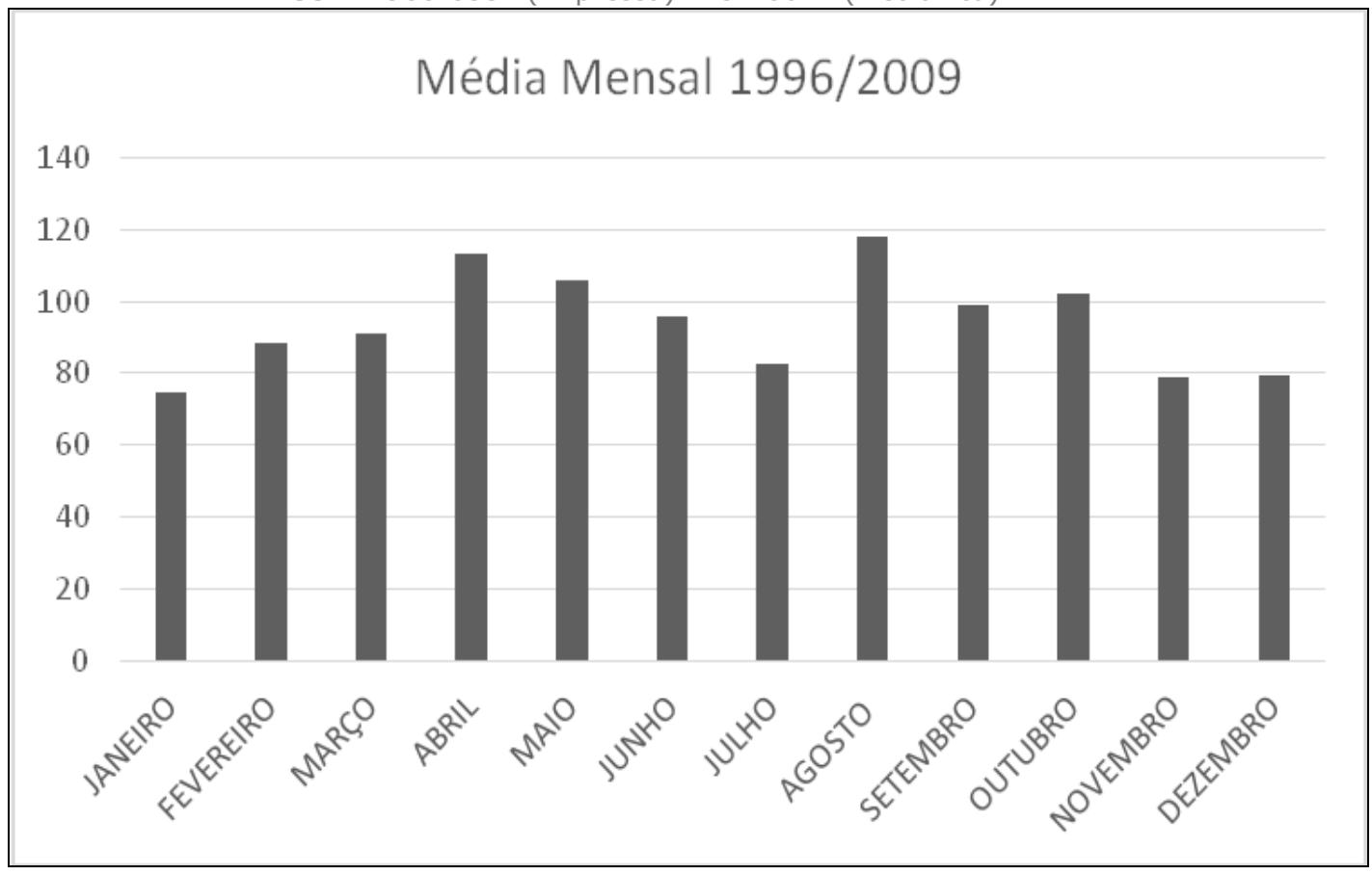

Figura 7 - Média Mensal na ESEC Taim 1996/2009. Org.: Simioni; Wollmann (2014).

\subsection{Média dos dias com chuva}

A média dos dias com chuva (figura 8) foi calculada a partir da média de chuva mensal dividida pelo número de horas no dia ( 24 horas) de cada um dos anos estudados. Deste modo, é possível identificar nesta figura que o mês de janeiro, mês de menor precipitação média anual, trimestral e mensal, também apresentou a menor média dos dias com chuva, ou seja, dentre os anos estudados o mês de janeiro teve uma média de apenas aproximadamente quatro dias com chuva.

Nos meses de outono e inverno os dias médios de chuva variam entre 6 a 6,5 dias com chuva, enquanto que no período de primavera e verão diminuem para aproximadamente 5 dias de chuvas durante os meses, atentando para uma maior disparidade no mês de outubro, o qual apresentou em média sete dias com precipitação pluviométrica, sendo então o mês de maior dias com chuva, se comparado a média dos outros meses. 


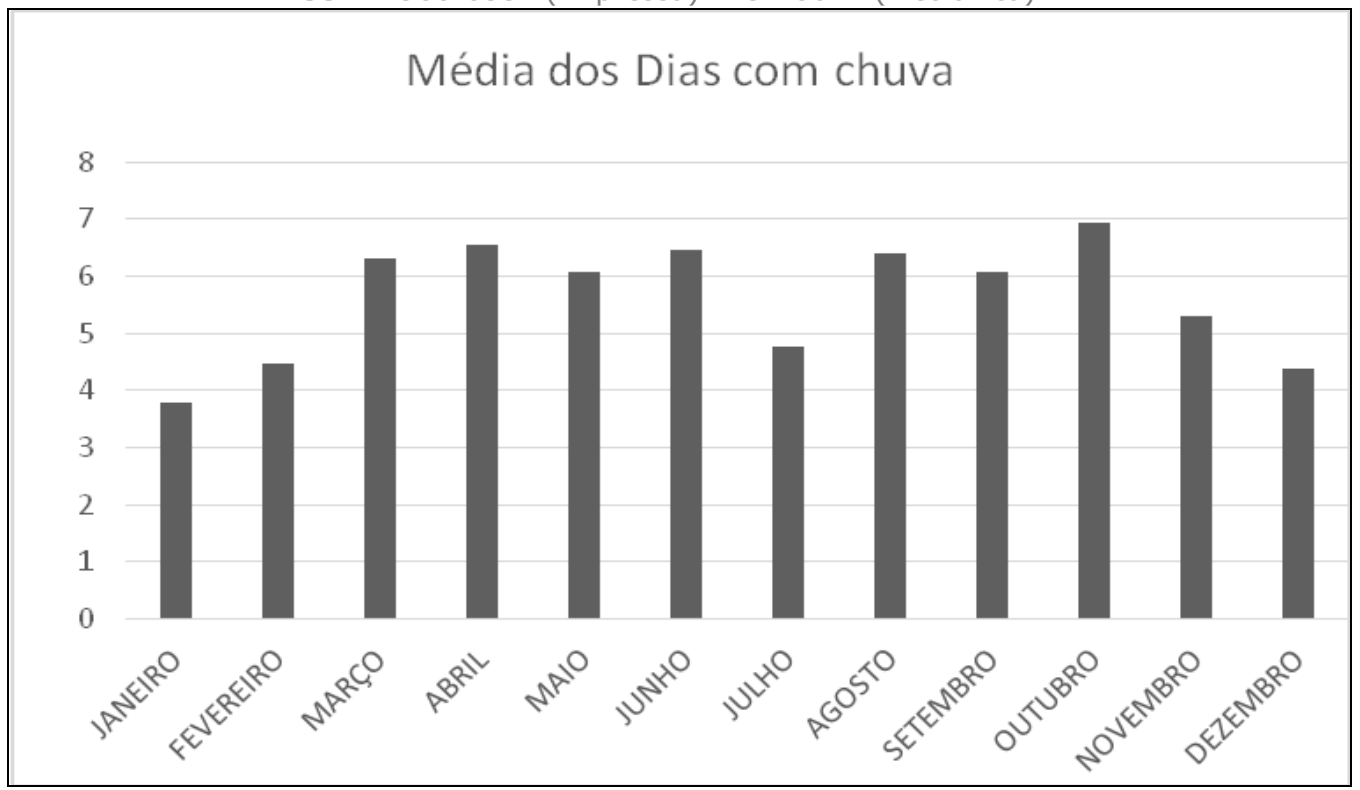

Figura 8 - Média mensal dos Dias com chuva na ESEC Taim 1996/2009 Org.: Simioni; Wollmann (2014).

\subsection{Variabilidade Interanual da Precipitação}

A figura 9 mostra a variabilidade interanual com desvios padrões positivos e negativos em relação ao desvio padrão para mais ou para menos entre os anos de 1996 a 2009. Através da aplicação da formula DP= PA - PM chegou-se a um valor de desvio padrão equivalente a 274,09 milímetros para mais ou para menos.

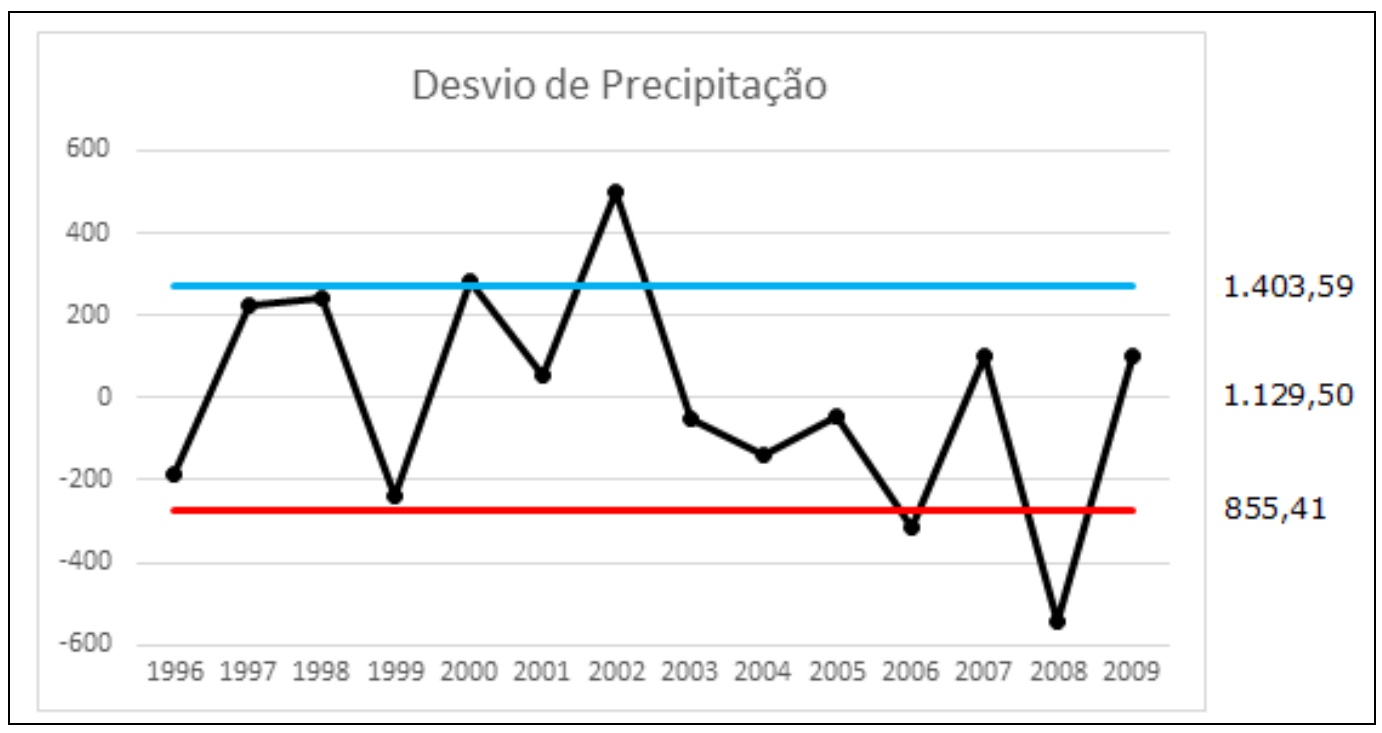

Figura 9 - Desvio interanual de precipitação na ESEC Taim. Org.: Simioni; Wollmann (2014). 
Assim sendo, considera-se dentro da normal climatológica dos anos estudados os valores do desvio médio de precipitação, que é $1.129,50$ milímetros mais ou menos 274,09, ou seja considera-se dentro da normal climatológica valores entre 1.403,59 milímetros e 855,41 milímetros.

Deste modo ao analisar a figura 4 verifica-se que a precipitação atingiu valores acima da normal climatológica para a Estação meteorológica do Taim nos anos 2000 e 2002, com 1413,85 mm e 1629,35 mm, respectivamente. Perto do desvio de precipitação máximo $(1.403,59 \mathrm{~mm})$ tem-se os anos de 1997 e 1998, onde a precipitação alcançou 1354,71 mm e 1374,69 mm, respectivamente.

Já aos valores mínimos, atingiram números inferiores a normal climatológica os anos de 2006 e 2008, com a precipitação máxima de 812,42 $\mathrm{mm}$ e $584,16 \mathrm{~mm}$, respectivamente.

Os anos que tiveram chuvas próximas ao desvio padrão mínimo, porém ainda dentro da normal climatológica foram os anos de 1996 e 1999, com exatos 945,7 e $892,78 \mathrm{~mm}$ de chuva, respectivamente.

\subsection{Comparação com Dados da Estação Meteorológica de Rio Grande e de Santa Vitória do Palmar/RS.}

A ESEC Taim é dividida por dois municípios, sendo sua parte norte pertencente ao município de Rio Grande e a porção sul do Taim pertencente ao município de Santa Vitória do Palmar. A Estação meteorológica do INMET em Rio Grande fica a aproximadamente $69 \mathrm{~km}$ de distância em linha reta da estação meteorológica do Taim. A Estação de Rio Grande fica a 2,6 metros de altitude em relação ao nível do mar, já a Estação do Taim fica a 2,1 metros do nível do mar.

Por outro lado, a estação meteorológica de Santa Vitória do Palmar fica a aproximadamente $134,0 \mathrm{~km}$, em linha reta, de distância da Estação meteorológica do Taim. A estação do INMET em Santa Vitória do Palmar fica a 24,5 metros de altitude, valor mais relevante se comparado as altitudes das estações do Taim e de Rio Grande.

Deste modo, verifica-se na figura 10 que, nos meses de janeiro e fevereiro a maior ocorrência de precipitação deu-se na estação meteorológica de Santa Vitória do Palmar, a estação de maior altitude entre as três estações em análise. Entre os meses de abril, maio, outubro, novembro e dezembro, choveu mais na estação pluviométrica do Taim, se comparado com a normal climatológica de Rio Grande. 


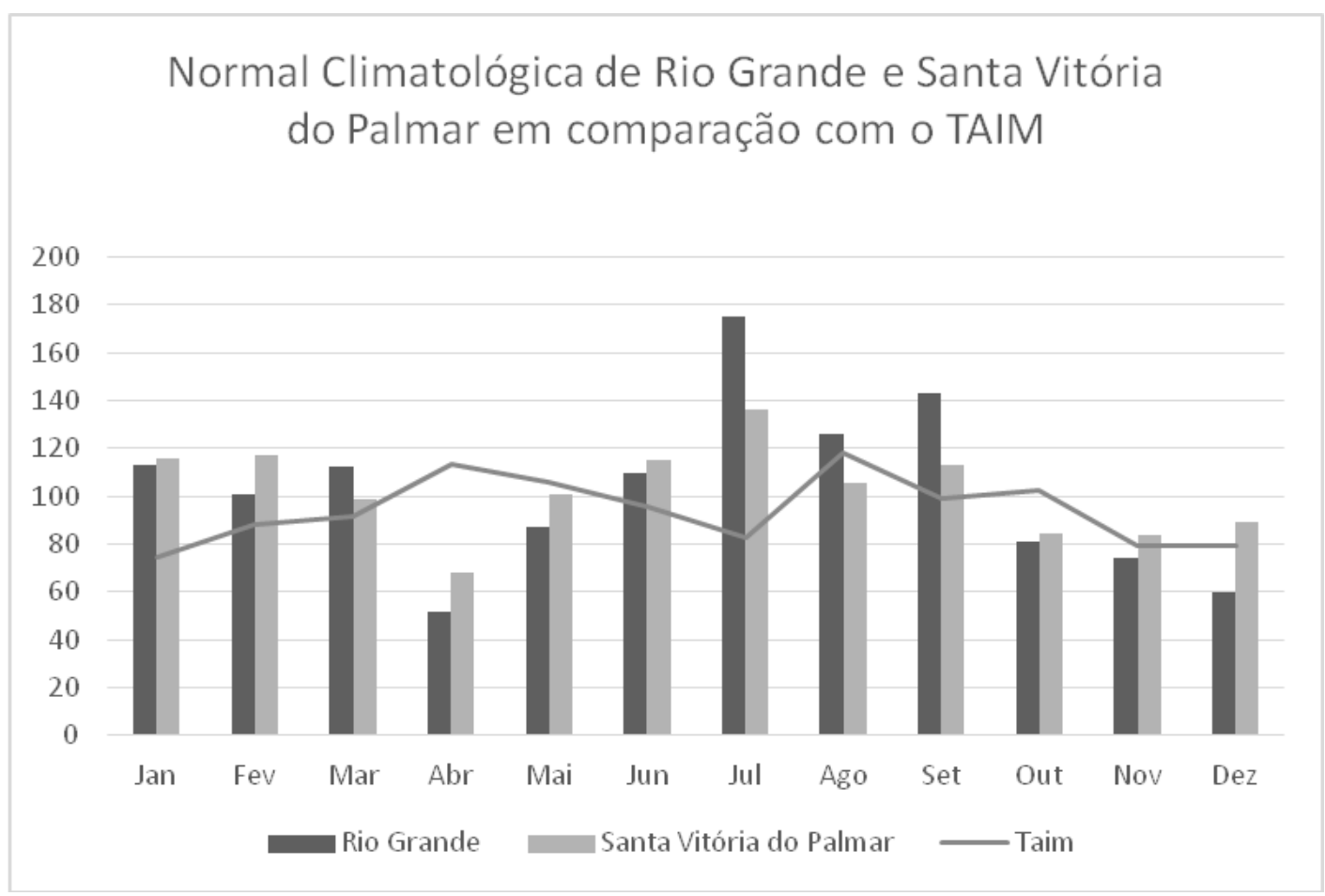

Figura 10 - Comparação com Dados da Estação Meteorológica de Rio Grande e de Santa Vitória do Palmar. Org.: Simioni; Wollmann (2014).

Por outro lado, a normal climatológica referente a Santa Vitória do Palmar revela que a precipitação no Taim foi superior apenas em abril, maio e outubro.

Atenta-se para a grande diferença entre as normais climatológicas de Rio Grande e do Taim no mês de julho. Sendo a normal para Rio Grande aproximadamente $170 \mathrm{~mm}$, enquanto que nos anos estudados a normal climatológica no Taim ficou na casa dos 80 milímetros, ou seja, a normal climatológica de Rio Grande é mais que o dobro da normal do Taim para o mês de julho.

\section{CONCLUSÕES}

Com a realização deste modesto trabalho de caracterização e variabilidade interanual da precipitação pluvial na ESEC Taim, almeja-se primeiramente, poder humildemente iniciar a contribuição científica do clima para o estudo das Unidades de Conservação (UC) brasileiras, especialmente as localizadas no Estado do Rio Grande do Sul, como a Estação Ecológica do Taim, de grande importância para a biota, mas ao mesmo tempo, frágil. 
Ainda, no âmago das Unidades de Conservação, o monitoramento climático das mesmas ainda constitui-se em ramo científico pouco estudado no Brasil, o que pode auxiliar significativamente no Plano de Manejo das UCs, não só na sua construção, bem como na sua plena execução de monitoramento.

A variável precipitação pluviométrica verificada entre os doze anos estudados demonstrou ser bem distribuída sazonalmente, sendo os meses de abril, maio, agosto, setembro e outubro os meses de maior precipitação pluviométrica para a ESEC Taim, enquanto que os meses de janeiro, julho, novembro e dezembro apresentam as menores médias de precipitação pluviométrica mensal.

Esta variabilidade mensal, não permite criar um modelo climático sazonal para a ESEC Taim, pois a variável precipitação pluviométrica é tão diversa quanto a biota existente no local, onde tratando-se de sazonalidade apenas o verão é entendido como um período de baixa precipitação, já os outros meses em questão apresentam uma variação bastante oscilatória, com meses de baixo regime pluviométrico e meses de alto índice de chuva.

Ao comparar os dados da ESEC Taim com os dados da Estação meteorológica de Rio Grande e de Santa Vitoria do Palmar nota-se no verão o índice de precipitação pluviométrica é menor no Taim se comparado ao das estações das cidades vizinhas, no entanto no outono este valor se inverte e o Taim apresenta um maior regime pluviométrico.

Já no inverno a precipitação na ESEC Taim volta a ser menor se comparada a de Rio Grande e Santa Vitoria do Palmar, invertendo novamente na primavera, ou seja, no verão e inverno a maior precipitação é percebida nas cidades vizinhas ao Taim e no outono e primavera chove mais na ESEC Taim do que em Rio Grande e Santa Vitoria do Palmar.

\section{REFERÊNCIAS BIBLIOGRÁFICAS}

ARMANI, G.; GALVANI, E. Avaliação do desempenho de um abrigo meteorológico de baixo custo. Revista Brasileira de Agrometeorologia, Piracicaba-SP, v. 14, n.1, p. 116-122, 2006.

BEZERRA, R. B.; DANTAS, R. T.; TRINDADE, A. G. Temporary characterization of the rainfall in Porto Velho/RO in the period of 1945 to 2003. Sociedade e Natura. Vol. 22. 2010. p. 609-623

DECRETO FEDERAL n 92.963, de 21 de Julho de 1986.

FLOOR, A. S. Estação Ecológica do Taim. 1999. Disponível em: <http://www.arroiogrande.com/especiais_estacaoecologicadotaim.htm> Acesso em 09 de março de 2012.

FUNDAÇÃO ZOOBOTÂNICA DO RIO GRANDE DO SUL (FZBRS). Mapa das Ecorregiões do Rio Grande do Sul. Disponível em: 
<http://www.biodiversidade.rs.gov.br/arquivos/1162476674ecorreg_rs.jpg> Acesso em: 05 de março de 2012.

KOURY, C. G.; RIZZO, E.; FREITAS, C.; ET. AL. Inclusão de Discussões de Mudanças Climáticas na Gestão de Unidades de Conservação. Manaus: Instituto de Conservação e Desenvolvimento Sustentável do Amazonas, 2011.

LIMA, N. G. B. Análise Microclimática dos Manguezais da Barra do Ribeira-Iguape/SP. Dissertação de Mestrado (Mestrado em Geografia Física). Universidade de São Paulo. São Paulo, 2009. 203p.

MILANESI, M. A.; GALVANI, E. Pluviômetro Experimental Para Localidades Remotas. In.: Climatologia Aplicada: Resgate aos Estudos de Caso. Galvani, E.; Lima, N. G. B. (Orgs.). Curitiba: Editora CRV, 2012. v. 1. 192 p. 1 ed.

MINISTÉRIO DO MEIO AMBIENTE (MMA). Mapa Ilustrativo do Sistema Nacional de Unidades de Conservação da Natureza. 2011. Disponível em: <http://www.ambientebrasil.com.br/images/mapas/uc_todas.pdf> Acesso em: 08 de março de 2012.

PANCOTTO, L. P. Influência Dos Eventos Oceano Climáticos Na Costa Sul Do Brasil. In.: ENCONTRO ESTADUAL DE GEOGRAFIA. 27. 2007, Santa Maria. Anais... Santa Maria: UNIFRA, 2007.

ROSSATO, M. S. Os Climas do Rio Grande do Sul: variabilidade, Tendências e Tipologia. Tese (Doutorado em Geografia). Universidade Federal do Rio Grande do Sul, Porto Alegre, 2011, $253 p$.

SARTORI, M. G. B. Distribuição das chuvas no Rio Grande do Sul e a variabilidade têmporoespacial no período 1912-1984. In: SIMPÓSIO BRASILEIRO DE GEOGRAFIA FÍSICA E APLICADA. 5. 1993, São Paulo. Anais... São Paulo: USP, 1993.

SEMC - SECRETARIA ESTADUAL DE MINAS, ENERGIA E COMUNICAÇÕES. Governo do Estado do Rio Grande do Sul. Atlas Eólico do Rio Grande do Sul. Porto Alegre, 2002. 65p.

SERAFINI JR., S. O Microclima de uma Vereda Degradada: Estudo de Caso no Parque Estadual Veredas do Peruaçu - MG. Tese (Doutorado em Geografia Física). Universidade de São Paulo. São Paulo, 2010. 140p.

SISTEMA NACIONAL DE UNIDADES DE CONSERVAÇÃO (SNUC). Lei No 9.985, de 18 de Julho de 2000.

WOLLMANN, C. A. Zoneamento Agroclimático para a Produção de Roseiras (Rosaceae spp.) no Rio grande do Sul. Tese (Doutorado em Geografia Física). Universidade de São Paulo. São Paulo, 2011, 386p. 2v.

WOLLMANN, C. A.; SARTORI, M. G. B. O Clima do Rio Grande do Sul No Verão: Análise Sobre a Circulação Atmosférica Regional e os Principais Tipos de Sucessão do Tempo em Três Casos Típicos. Revista Geografia Ensino e Pesquisa, v. 13, p. 33-43, 2009a.

WOLLMANN, C. A.; SARTORI, M. G. B. Frequência mensal e sazonal da participação de sistemas atmosféricos no verão do Rio Grande do Sul: análise sobre três casos típicos (1986/1987, 1997/1998 e 2004/2005). Revista Ciência e Natura, v. 31, p. 141-161, 2009b.

WOLLMANN, C. A.; SIMIONI, J. P. D. Variabilidade espacial dos atributos climáticos na Estação Ecológica do Taim (RS), sob domínio polar. Revista do Departamento de Geografia (USP). Volume 25, 2013. p. 56-76. 